\title{
Conversion des valeurs génétiques des taureaux laitiers nord-américains en index français
}

\author{
B. BONAÏTI et P. BOULANGER * \\ INRA, Station de Génétique Quantitative et Appliquée \\ Centre de Recherches Zootechniques, 78350 Jouy-en-Josas, France \\ * Institut Technique de l'Elevage Bovin \\ 149, rue de Bercy, 75595 Paris Cedex 12, France
}

\begin{abstract}
Résumé
Une analyse des performances en France des filles de taureaux d'origine nord-américaine donne des formules permettant de convertir les valeurs génétiques publiées aux Etats-Unis ou au Canada selon le système français d'évaluation. La méthode, déduite de celle décrite par GodDarD (1985), s'appuie sur la régression des déviations moyennes des filles des taureaux sur leur valeur génétique en Amérique du Nord. Deux groupes de données sont distingués selon que la valeur génétique du taureau est estimée à partir de sa descendance en Amérique du Nord ou des valeurs génétiques de son père et de son grand-père maternel. Des formules sont données pour les trois critères utilisés en France pour l'évaluation des taureaux : la quantité moyenne de matière utile (MMU), le taux moyen de matière utile (MTX) et la quantité de lait. C'est seulement pour la conversion entre les Etats-Unis et la France que des formules précises peuvent être données, la taille des fichiers n'étant pas suffisante dans le cas du Canada. Les corrélations obtenues entre les valeurs génétiques estimées aux Etats-Unis et les performances moyennes des filles des mêmes taureaux en France n'excluent pas l'existence d'une interaction génotype-milieu entre ces deux pays. Celle-ci resterait cependant assez limitée pour permettre l'utilisation des formules de conversion.
\end{abstract}

Mots clés : valeur génétique, bovins laitiers, conversion entre pays, lait, matière grasse, matière protéique.

\section{Summary}

Conversion of North American dairy sire proofs into French breeding values

Formulae converting US and Canadian sire proofs into French breeding values were obtained from an analysis of records of French daughters sired from bulls born or out of parents born in North America. The method, derived from GodDard (1985), is based upon the regression of mean daughter deviations on sire evaluation in North America. Two data sets were considered depending on whether the American sire breeding values were estimated from progeny test results in North America or from pedigree information (sire and maternal grand sire). Formulae are given for the three variables used in French evaluation : useful matter yield (MMU) or content (MTX) and milk yield. Due to the limited size of the Canadian data set, precise formulae can only be given for conversion between US predicted difference (PD) and French breeding value (IF) (using respectively the 1982 and 1985 bases). 


$$
\begin{aligned}
& \mathrm{IF}=944+0.76 \mathrm{PD} \text { for milk yield } \\
& \mathrm{IF}=27+0.76(\text { PDfat }+1.15 \text { PDprotein }) / 2 \text { for MMU } \\
& \mathrm{IF}=-1.6+26(\text { PDfat }+1.15 \text { PDprotein }) / 2 \text { for MTX }
\end{aligned}
$$

Correlations between US breeding values and daughter performances in France do not exclude any genotype-environment interaction between the two countries. However this interaction would not be large enough to prevent the use of the conversion formulae.

Key words : breeding value, dairy cattle, conversion between countries, milk, fat, protein.

\section{Introduction}

Les échanges de reproducteurs mâles entre pays se sont largement développés au cours des dix dernières années. En France, parmi les taureaux de race Frisonne mis en testage sur descendance, la part de ceux issus de parents américains est devenue prépondérante. Des taureaux déjà testés en Amérique du Nord sont également utilisés pour la production de génisses ou de reproducteurs mâles. Une telle évolution nécessite que les reproducteurs, dont la valeur génétique fut obtenue aux Etats-Unis ou au Canada, puissent être replacés avec précision parmi l'éventail français de variabilité génétique. Ceci rejoint les objectifs formulés par la Fédération Internationale Laitière (IDF) qui propose une formule de conversion permettant de traduire la valeur génétique $\left(\mathrm{G}_{\mathrm{o}}\right)$ obtenue dans le pays d'origine en une valeur équivalente pour le pays importateur $\left(G_{f}\right)$ (GRAVERT, 1983).

$$
\mathrm{G}_{\mathrm{i}}=\mathbf{a}+\mathbf{b} \mathbf{G}_{\mathrm{o}}
$$

L'analyse des performances en France des filles de taureaux dont la valeur génétique est connue également aux Etats-Unis ou au Canada (sur ascendance ou descendance) permet d'estimer les paramètres $a$ et $b$ nécessaires à la transformation des valeurs génétiques nord-américaines en équivalents français pour les trois principaux critères de sélection utilisés en France : la quantité de lait, la quantité et le taux de matière utile (MMU et MTX).

\section{Matériel et méthodes}

\section{A. Matériel animal}

Deux sources d'information différentes sont disponibles. La première est relative à des taureaux utilisés en France après un testage sur descendance en Amérique du Nord (importation de semences). Ces taureaux sont choisis selon leur valeur génétique estimée en Amérique du Nord par des éleveurs désireux d'accroître très rapidement le potentiel génétique de leur troupeau. La seconde source d'information résulte de l'importation, par les unités de sélection françaises, de mâles déjà connus en Amérique du Nord selon l'ascendance, qui sont testés en France sur descendance. Ces deux sources d'information seront, par la suite, respectivement nommées sous l'appellation de groupe I (« semences importées») ou II («taureaux importés»). 


\section{Taureaux issus des Etats-Unis}

En 1985, on dispose, pour le groupe I, de 90 taureaux, de valeurs génétiques publiées par l'USDA (United States Department of Agriculture), qui ont un minimum de 35 filles en France. Les informations nord-américaines proviennent des publications de l'USDA postérieures à 1976, 63 taureaux ayant un index publié en 1981 ou après. Le dernier index publié est retenu pour chaque taureau. La demi-valeur génétique moyenne des taureaux (la valeur génétique transmise) est, vis-à-vis de la base 1982 des Etats-Unis, de -38 livres de lait, +4 livres de matières grasses et $+0,04 \%$ de taux butyreux (tableau 1). Seuls 67 taureaux sont connus pour la matière azotée et ont un index moyen de +1 livre de matière azotée et de $+0,00 \%$ de taux azoté. La répétabilité moyenne des index des taureaux est de 0,98 pour les quantités de lait et de matière grasse et de 0,87 pour la matière azotée.

\section{TABleaU 1}

Valeurs génétiques des taureaux issus des Etats-Unis Breeding values of bulls from United States

\begin{tabular}{|c|c|c|c|c|c|c|c|c|c|c|c|}
\hline \multirow{3}{*}{$\begin{array}{c}\text { Grou- } \\
\text { pe }\end{array}$} & \multirow{3}{*}{$\mathbf{n}$} & \multicolumn{6}{|c|}{ Résultats aux Etats-Unis } & \multicolumn{4}{|c|}{ Résultats en France ${ }^{* * *}$} \\
\hline & & \multirow[t]{2}{*}{ Variable } & \multirow[t]{2}{*}{$\mathrm{CD}$} & \multicolumn{2}{|c|}{$\begin{array}{l}\text { Unités * } \\
\text { des USA }\end{array}$} & \multicolumn{2}{|c|}{$\begin{array}{l}\text { Unités ** } \\
\text { françaises }\end{array}$} & \multirow[t]{2}{*}{ Variable } & \multirow[t]{2}{*}{$\mathrm{CD}$} & \multirow[t]{2}{*}{$\mathbf{m}$} & \multirow[t]{2}{*}{$\sigma$} \\
\hline & & & & $\mathbf{m}$ & $\boldsymbol{\sigma}$ & m & $\boldsymbol{\sigma}$ & & & & \\
\hline $\begin{array}{l}\text { I } \\
\text { II }\end{array}$ & $\begin{array}{r}90 \\
649\end{array}$ & Lait & $\begin{array}{l}98 \\
31\end{array}$ & $\begin{array}{l}-38 \\
-296\end{array}$ & $\begin{array}{l}517 \\
304\end{array}$ & $\begin{array}{l}-34 \\
-266\end{array}$ & $\begin{array}{l}465 \\
274\end{array}$ & Lait & $\begin{array}{l}85 \\
86\end{array}$ & $\begin{array}{r}1179 \\
723\end{array}$ & $\begin{array}{l}504 \\
630\end{array}$ \\
\hline $\begin{array}{l}\text { I } \\
\text { II }\end{array}$ & $\begin{array}{r}67 \\
429\end{array}$ & Mat. azotée (MA) & $\begin{array}{l}87 \\
29\end{array}$ & $\begin{array}{r}1 \\
-4\end{array}$ & $\begin{array}{r}15 \\
7\end{array}$ & $\begin{array}{r}1 \\
-4\end{array}$ & $\begin{array}{r}14 \\
6\end{array}$ & & & & \\
\hline $\begin{array}{l}\text { I } \\
\text { II }\end{array}$ & $\begin{array}{r}90 \\
649\end{array}$ & Mat. grasse (MG) & $\begin{array}{l}98 \\
31\end{array}$ & $\begin{array}{r}4 \\
-4\end{array}$ & $\begin{array}{l}18 \\
11\end{array}$ & $\begin{array}{r}4 \\
-3\end{array}$ & $\begin{array}{l}16 \\
10\end{array}$ & MMU & $\begin{array}{l}85 \\
86\end{array}$ & $\begin{array}{l}36 \\
23\end{array}$ & $\begin{array}{l}20 \\
19\end{array}$ \\
\hline $\begin{array}{l}\text { I } \\
\text { II }\end{array}$ & $\begin{array}{r}67 \\
429\end{array}$ & Taux azoté (TA) & & $\begin{array}{l}0,00 \\
0,02\end{array}$ & $\begin{array}{l}0,06 \\
0,03\end{array}$ & $\begin{array}{l}0,1 \\
0,4\end{array}$ & $\begin{array}{l}1,2 \\
0,6\end{array}$ & & & & \\
\hline $\begin{array}{l}\text { I } \\
\text { II }\end{array}$ & $\begin{array}{r}90 \\
649\end{array}$ & Taux butyreux (TB) & & $\begin{array}{l}0,04 \\
0,05\end{array}$ & $\begin{array}{l}0,12 \\
0,05\end{array}$ & $\begin{array}{l}0,8 \\
0,9\end{array}$ & $\begin{array}{l}2,4 \\
0,9\end{array}$ & MTX & $\begin{array}{l}91 \\
92\end{array}$ & $\begin{array}{l}-1,1 \\
-0,6\end{array}$ & $\begin{array}{l}2,2 \\
2,3\end{array}$ \\
\hline
\end{tabular}

* Valeur génétique transmise en livres (lait, MA ou MG) ou \% (TA, TB) vis-à-vis de la base 1982 des EtatsUnis.

** Valeur génétique en $\mathrm{kg}$ (lait, MA ou MG) ou g/kg (TA ou TB) vis-à-vis de la base 1982 des Etats-Unis.

*** $2 \times$ déviation des filles en kg (lait, MMU) ou g/kg (MTX) vis-à-vis de la base 1985 de la France.

* Transmitted breeding value in pounds (milk, protein or fat) or percent (protein or fat percent) as deviation from the base of 1982.

** Breeding value in $\mathrm{kg}$ (milk, protein or fat) or $\mathrm{g} / \mathrm{kg}$ (protein or fat percent) as deviations from the base of 1982.

*** $2 \times$ daughters deviation in $\mathrm{kg}$ (milk, MMU) or $\mathrm{g} / \mathrm{kg}(M T X)$ as deviation from the base of 1985.

n : nombre de taureaux / number of bulls.

$\mathrm{CD}$ : coefficient de détermination / repeatability.

m : moyenne / mean.

$\sigma$ : écart-type / standard deviation.

Groupe I : taureaux utilisés en France après testage en Amérique du Nord.

bulls used in France after progeny testing in North America.

Groupe II : taureaux issus de parents américains et testés en France.

bulls out of parents born in North America and proved in France.

$\mathrm{MMU}=($ Fat yield +1.21 Protein yield $) / 2$.

MTX = MMU/Milk. 
Par ailleurs, entre l'introduction des premiers taureaux Holstein et 1985, les centres d'insémination français ont testé 1084 taureaux issus de pères enregistrés aux EtatsUnis. Parmi ceux-ci, seuls 649 taureaux respectent les conditions suivantes :

- naissance ou procréation aux Etats-Unis après 1971 ;

- père et grand-père maternel connus aux Etats-Unis avec un coefficient de répétabilité supérieur ou égal à 0,90 pour la quantité de matière grasse.

Parmi ces derniers, seuls 429 taureaux ont leurs père et grand-père maternel également connus pour la matière azotée avec un coefficient de répétabilité au moins égal à 0,60 . Cet ensemble des 649 taureaux (seulement 429 pour les analyses faisant intervenir la matière azotée) est retenu pour le groupe II. En tenant compte des valeurs génétiques du père et du grand-père maternel, les 649 taureaux ont une valeur génétique transmise de -296 livres de lait, -4 livres de matière grasse et $+0,05 \%$ de taux butyreux. Le sous-ensemble de 429 taureaux se situe à -4 livres de matière azotée et $+0,02 \%$ de taux azoté (tableau 1 ).

\section{Taureaux issus du Canada}

Le groupe de taureaux canadiens de type I est constitué de 52 taureaux ayant au minimum 15 filles contrôlées en France. Selon les publications canadiennes (1978 à 1984), ces taureaux ont, en moyenne et vis-à-vis de la base canadienne 1984, des valeurs génétiques respectives de $-0,4,-2,4$ points $\mathrm{BCA}$ et $-0,06 \%$ pour le lait, la matière grasse et le taux butyreux (tableau 2) (le point BCA, unité utilisée au Canada pour l'évaluation des taureaux laitiers, correspond respectivement à $53 \mathrm{~kg}, 1,96 \mathrm{~kg}$ et $1,68 \mathrm{~kg}$ de lait, de matière grasse et de matière azotée - DAIRY SIRE APPRAISAL, 1985). Parmi ceux-ci, 45 taureaux sont également connus pour la matière azotée et ont une valeur de $-1,1$ point $\mathrm{BCA}$ pour la quantité et de $-0,02 \%$ pour le taux. Ces taureaux ont un coefficient de détermination moyen de 0,95 pour la matière grasse et de 0,93 pour la matière azotée.

Le groupe de taureaux de type II est constitué de 52 taureaux dont les père et grand-père maternel sont chacun connus au Canada avec des coefficients de détermination supérieurs à 0,90 pour la matière grasse. Leur valeur génétique sur ascendance (père et grand-père maternel) est en moyenne de $-2,9$ et $-3,4$ points pour le lait et la matière grasse et de $-0,01 \%$ pour le taux butyreux. Parmi ceux-ci, 40 taureaux, connus pour la matière azotée, ont une valeur moyenne sur ascendance de $-2,7$ points pour la quantité et de $-0,01 \%$ pour le taux.

\section{B. Méthodes}

GodDard (1985) propose une estimation des coefficients a et $b$ de la formule de conversion (1) à l'aide de la régression de la "valeur génétique estimée dérégressée » sur la valeur génétique estimée dans le pays d'origine. La seconde des méthodes proposées par Wilmink et al. (1986) repose sur le même principe. La «valeur génétique dérégressée " est en fait le double de la déviation moyenne des filles lorsque l'estimation de la valeur génétique est obtenue par une régression simple. Le système français de calcul des valeurs génétiques permet de disposer des déviations moyennes de performances corrigées pour l'ensemble des facteurs de variation du milieu. La méthode utilisée ici reprend donc le principe de GodDard (1985) tout en s'appuyant directement sur les déviations moyennes des taureaux. 
TABLEAU 2

Valeurs génétiques des taureaux issus du Canada Breeding values of bulls from Canada

\begin{tabular}{|c|c|c|c|c|c|c|c|c|c|c|c|}
\hline \multirow{3}{*}{$\begin{array}{c}\text { Grou- } \\
\text { pe }\end{array}$} & \multirow{3}{*}{$\mathbf{n}$} & \multicolumn{6}{|c|}{ Résultats au Canada } & \multicolumn{4}{|c|}{ Résultats en France ${ }^{* * *}$} \\
\hline & & \multirow[t]{2}{*}{ Variable } & \multirow[t]{2}{*}{ CD } & \multicolumn{2}{|c|}{$\begin{array}{c}\text { Unités * } \\
\text { canadiennes }\end{array}$} & \multicolumn{2}{|c|}{$\begin{array}{l}\text { Unités ** } \\
\text { françaises }\end{array}$} & \multirow[t]{2}{*}{ Variable } & \multirow[t]{2}{*}{$C D$} & \multirow[t]{2}{*}{$\mathrm{m}$} & \multirow[t]{2}{*}{$\boldsymbol{\sigma}$} \\
\hline & & & & $\mathbf{m}$ & $\sigma$ & $\mathrm{m}$ & $\boldsymbol{\sigma}$ & & & & \\
\hline $\begin{array}{l}\text { I } \\
\text { II }\end{array}$ & $\begin{array}{l}52 \\
52 \\
\end{array}$ & Lait & & $\begin{array}{l}-0,4 \\
-2,9 \\
\end{array}$ & $\begin{array}{l}4,8 \\
2,8 \\
\end{array}$ & $\begin{array}{r}-37 \\
-311 \\
\end{array}$ & $\begin{array}{l}512 \\
298 \\
\end{array}$ & Lait & $\begin{array}{l}74 \\
82 \\
\end{array}$ & $\begin{array}{l}510 \\
286 \\
\end{array}$ & $\begin{array}{l}641 \\
487 \\
\end{array}$ \\
\hline $\begin{array}{l}\text { I } \\
\text { II }\end{array}$ & $\begin{array}{l}45 \\
40 \\
\end{array}$ & Mat. azotée (MA) & $\begin{array}{l}93 \\
29 \\
\end{array}$ & $\begin{array}{l}-1,1 \\
-2,7\end{array}$ & $\begin{array}{l}5,0 \\
2,5 \\
\end{array}$ & $\begin{array}{l}-4 \\
-9 \\
\end{array}$ & $\begin{array}{r}17 \\
9 \\
\end{array}$ & & & & \\
\hline II & $\begin{array}{l}52 \\
52 \\
\end{array}$ & Mat. grasse (MG) & $\begin{array}{l}95 \\
30 \\
\end{array}$ & $\begin{array}{l}-2,4 \\
-3,4\end{array}$ & $\begin{array}{l}6,2 \\
3,7\end{array}$ & $\begin{array}{r}-9 \\
-13\end{array}$ & $\begin{array}{l}24 \\
14\end{array}$ & MMU & $\begin{array}{l}74 \\
82\end{array}$ & $\begin{array}{r}9 \\
10\end{array}$ & $\begin{array}{l}24 \\
19\end{array}$ \\
\hline $\begin{array}{l}\text { I } \\
\text { II }\end{array}$ & $\begin{array}{l}45 \\
40\end{array}$ & Taux azoté (TA) & & $\begin{array}{l}-0,02 \\
-0,01 \\
\end{array}$ & $\begin{array}{l}0,06 \\
0,04 \\
\end{array}$ & $\begin{array}{l}-0,5 \\
-0,2 \\
\end{array}$ & $\begin{array}{l}1,2 \\
0,8\end{array}$ & & & & \\
\hline I & $\begin{array}{l}52 \\
52\end{array}$ & Taux butyreux (TB) & & $\begin{array}{l}-0,06 \\
-0,01 \\
\end{array}$ & $\begin{array}{l}0,14 \\
0,07\end{array}$ & $\begin{array}{l}-1,2 \\
-0,3 \\
\end{array}$ & $\begin{array}{l}2,8 \\
1,4 \\
\end{array}$ & MTX & $\begin{array}{l}86 \\
90 \\
\end{array}$ & $\begin{array}{l}-1,6 \\
-0,6\end{array}$ & $\begin{array}{l}2,0 \\
2,0\end{array}$ \\
\hline
\end{tabular}

* Valeur génétique transmise en points BCA (lait, MA ou MG) ou \% (TA, TB) vis-à-vis de la base 1984 du Canada.

** Valeur génétique du taureau en $\mathrm{kg}$ (lait, MA ou MG) ou g/kg (TA ou TB) vis-à-vis de la base 1984 du Canada.

*** $2 \times$ déviation des filles en $\mathrm{kg}$ (lait, MMU) ou $\mathrm{g} / \mathrm{kg}$ (MTX) vis-à-vis de la base 1985 de la France.

* Transmitted breeding value in terms of BCA (milk, protein or fat) or percent (protein or fat percent) as deviations from the base of 1984.

** Breeding value in terms of $\mathrm{kg}$ (milk, protein or fat) or $\mathrm{g} / \mathrm{kg}$ (protein or fat percent) as deviation from the base of 1984.

*** $2 \times$ daughters deviation in terms of $\mathrm{kg}$ (milk, MMU) or $\mathrm{g} / \mathrm{kg}(\mathrm{MTX})$ as deviation from the base of 1985 .

$\mathrm{n}, \mathrm{CD}, \mathrm{m}, \sigma$, Groupe, MMU et MTX : voir tableau 1 / see table 1.

En suivant le principe proposé par Falconer (1952) pour l'étude de l'interaction génotype-milieu, on associe à tout reproducteur d'un échantillon de mâles de la population Frisonne du pays d'origine deux valeurs génétiques différentes $G_{f}$ et $G_{o}$ correspondant respectivement à la production laitière en France et dans le pays d'origine. Ces variables aléatoires sont reliées par une relation faisant intervenir une valeur génétique résiduelle $(\mathrm{g})$ indépendante de $\mathrm{G}_{\mathrm{o}}$ :

$$
\mathrm{G}_{\mathrm{f}}=\mathrm{a}+\mathrm{b} \mathrm{G}_{\mathrm{o}}+\mathrm{g} \text {. }
$$

Le modèle (2) admet implicitement la possibilité d'une interaction génotype-milieu correspondant à une modification du classement des génotypes entre eux et/ou à celle de l'amplitude des différences phénotypiques entre génotypes (paramètre b). La corrélation $R_{g}$ entre $G_{f}$ et $G_{o}$ est équivalente à une corrélation génétique. L'une et l'autre des deux formes d'interaction peuvent résulter de divers facteurs liés au milieu (alimentation, élevage, traite...) mais aussi de la nature précise des variables considérées pour l'évaluation de la valeur génétique : production totale corrigée pour la durée de traite 
en France ou production en 305 jours en Amérique, production observée sur la première, les trois premières ou toutes les lactations respectivement au Canada, en France et aux Etats-Unis. En outre, les données utilisées dans cette étude ne permettent pas d'éviter une confusion de l'interaction génotype-milieu avec d'éventuels effets d'hétérosis entre la souche Holstein d'Amérique du Nord et la population Frisonne Française.

Selon le modèle (2), la valeur génétique en France $\left(G_{t}\right)$ d'un taureau connu dans le pays d'origine $\left(\hat{G}_{0}\right)$ peut être estimée selon une formule identique à celle recommandée par l'IDF (1981) :

$$
\hat{G}_{\mathrm{f}}=E\left(G_{\mathrm{f}} / \hat{G}_{\mathrm{o}}\right)=a+b \hat{G}_{0} .
$$

L'estimation des paramètres a et b du modèle (2), qui permet donc d'obtenir la formule de conversion (1) de l'IDF (1981), repose sur l'analyse des liaisons entre la valeur génétique estimée d'un taureau dans son pays d'origine $\left(\hat{G}_{\mathrm{o}}\right)$ et la déviation moyenne des performances de ses filles en France $(M)$. La valeur génétique $\left(\hat{G}_{\mathrm{o}}\right)$ est un indice sur descendance pour les données du groupe I (semences importées) ou un indice sur ascendance (père et grand-père maternel) pour celles du groupe II (taureaux importés). Dans les deux groupes, $\hat{\mathbf{G}}_{0}$, qui résulte des données du pays d'origine uniquement, a un coefficient de détermination $R_{\mathrm{o}}^{2}$ obtenu directement dans les catalogues de valeurs génétiques (groupe I) ou calculé à partir de ceux de son père et de son grand-père maternel (groupe II). La déviation moyenne des filles (M) est utilisée en France pour le calcul d'une valeur génétique estimée et peut aussi être associée à un coefficient de détermination $R_{f}^{2}$ obtenu dans les publications d'index françaises. Le coefficient de régression $\beta\left(M / \hat{G}_{o}\right)$, la corrélation $r\left(M, \hat{G}_{0}\right)$ et l'espérance $E(M)$ dépendent des paramètres $a$, $b$ et $R_{g}$ du modèle 2 , des deux coefficients $R_{o}^{2}$ et $R_{i}^{2}$ et de la moyenne des valeurs génétiques estimées $\left(\hat{G}_{0}\right)$ dans l'échantillon de données analysées :

$$
\begin{aligned}
& \beta\left(M / \hat{G}_{o}\right)=b / 2 \\
& r\left(M, \hat{G}_{o}\right)=R_{o} R_{t} R_{g} \\
& E(M)=\left(a+b \hat{G}_{o}\right) / 2
\end{aligned}
$$

On en déduit :

$$
\begin{aligned}
& \hat{b}=2 \beta\left(M / \hat{G}_{o}\right) \\
& \hat{a}=2 \ddot{M}-\hat{b} \hat{G}_{o}
\end{aligned}
$$

et

$$
\hat{\mathbf{R}}_{\mathrm{g}}=\mathrm{r}\left(\mathbf{M}, \hat{\mathrm{G}}_{\mathrm{o}}\right) /\left(\mathrm{R}_{\mathrm{o}} \mathbf{R}_{\mathrm{f}}\right) \text {. }
$$

La qualité de ces estimations est conditionnée par celle de l'échantillon des reproducteurs mâles. Les taureaux (groupe I) ou leurs parents (groupe II) ont été sélectionnés selon leur valeur génétique estimée en Amérique du Nord. Ceci peut réduire l'estimation de la corrélation génétique $\left(R_{g}\right)$ mais ne modifie pas les estimations des paramètres a et $b$ liées à la régression de $\mathbf{M}$ sur $\hat{G}_{0}$ puisqu'aucune sélection n'a été exercée sur la variable $M$.

\section{Variables}

Les modes d'expression des valeurs génétiques diffèrent entre l'Amérique du Nord et la France. Les résultats concernent dans le premier cas la valeur transmise à la descendance (soit la demi-valeur génétique) et dans le second la valeur génétique du 
taureau. Les unités ne sont pas identiques. Les quantités sont données en livres aux Etats-Unis et en points BCA au Canada. La richesse du lait est présentée en $\%$ en Amérique du Nord et en $\mathrm{g} / \mathrm{kg}$ en France. Pour transformer les résultats nordaméricains selon le mode d'expression de la France, les quantités des Etats-Unis sont donc multipliées par 0,9, celles du Canada par 106, 3,92 et 3,36 respectivement pour les trois quantités de lait, de matières grasse et azotée ; les taux sont, dans les deux cas, multipliés par 20 .

Les taureaux ne sont connus en France que pour trois variables : la quantité de lait (L), la quantité et le taux de matière utile (MMU et MTX). Les deux dernières variables sont définies à partir des matières grasse (MG) et protéique (MP), et des taux butyreux (TB) et protéique (TP) selon les deux formules :

$$
\begin{aligned}
& \mathrm{MMU}=(\mathrm{MG}+1,21 \mathrm{MP}) / 2 \\
& \mathrm{MTX}=(\mathrm{TB}+1,21 \mathrm{TP}) / 2 .
\end{aligned}
$$

Des équivalents sont obtenus pour ces deux variables à partir des résultats nordaméricains selon les mêmes formules mais avec un coefficient de 1,15 au lieu de 1,21 pour la matière ou le taux azoté (MA ou TA) afin de tenir compte du fait que les résultats nord-américains et français sont relatifs à la matière azotée pour les premiers et à la matière protéique pour les seconds $(5 \%$ de l'azote total du lait étant non protéique ; GourSAUd, 1985).

Les valeurs génétiques estimées selon l'ascendance $\left(\hat{G}_{\mathrm{a}}\right)$ et les coefficients de détermination correspondants $\left(R_{\mathrm{a}}^{2}\right)$ sont déduits des formules suivantes :

$$
\begin{aligned}
& \hat{\mathrm{G}}_{\mathrm{a}}=0,5 \hat{\mathrm{G}}_{\mathrm{p}}+0,25 \hat{\mathrm{G}}_{\mathrm{gpm}} \\
& \mathrm{R}_{\mathrm{a}}^{2}=0,25 \mathrm{R}_{\mathrm{p}}^{2}+0,0625 \mathrm{R}_{\mathrm{gpm}}^{2}
\end{aligned}
$$

où $\hat{G}_{\mathrm{p}}$ et $\hat{\mathrm{G}}_{\mathrm{gpm}}$ sont les valeurs génétiques du père et du grand-père maternel et $\mathbf{R}_{\mathrm{p}}^{2}$, $\mathbf{R}_{\mathrm{gpm}}^{2}$ les coefficients de détermination correspondants.

Les valeurs génétiques américaines sont comparées à des déviations moyennes par taureau de performances françaises corrigées selon les principes définis pour le calcul des valeurs génétiques (PouTous et al., 1981; BonAïrI et al., 1984). Elles sont indépendantes de la valeur génétique des mères (grâce à une correction des données en fonction de la valeur génétique de celles-ci ) et des facteurs de variation liés au milieu (année, troupeau... ) ou à la vache (âge, mois ou rang de vêlage). Les données utilisées, qui peuvent être des lactations de rang 1, 2 ou 3 ont, du fait de différentes corrections multiplicatives préliminaires, une variabilité proche de celles de premières lactations non corrigées pour la durée. Les méthodes de calcul des valeurs génétiques françaises font intervenir un groupe de référence fixe composé de taureaux dont la première fille a eu un vêlage de rang inférieur ou égal à 3 en 1973 ou 1974. Par suite de toutes les corrections, en particulier celles liées aux effets de l'année, les déviations moyennes par taureau sont établies en écart à ce groupe de référence. Cependant, les valeurs génétiques françaises sont publiées sous forme d'écarts à une base mobile, c'està-dire à la moyenne pondérée des valeurs génétiques des taureaux des 4 dernières séries, sachant que la série d'un taureau est définie par la campagne de premier vélage de sa première fille. Pour obtenir des formules de conversion relatives à la base mobile 1985, les déviations moyennes ont été diminuées de la moitié de la valeur de celle-ci, la base 1985 étant égale à $33 \mathrm{~kg}$ de $\mathrm{MMU}, 901 \mathrm{~kg}$ de lait et $-0,01 \mathrm{~g} / \mathrm{kg}$ de MTX. 


\section{Résultats}

\section{A. Comparaison avec les Etats-Unis}

Les estimations obtenues pour les différents paramètres sont rassemblées dans le tableau 3. La précision d'estimation des paramètres $a$ et $b$ est équivalente entre les deux groupes de données. La bonne précision résulte dans le premier groupe de l'association élevée qui existe entre la valeur génétique américaine du taureau et la moyenne de ses filles en France et dans le second groupe du plus grand effectif de couples de données. La précision d'estimation ne peut donc être invoquée pour rejeter ou privilégier l'une de ces deux sources d'information.

TABLEAU 3

Relations entre les valeurs génétiques * aux Etats-Unis et en France Relationship between breeding values * in USA and France

\begin{tabular}{|c|c|c|c|c|c|c|}
\hline Variable & Groupe & b & a & $r\left(\mathbf{M}, \hat{G}_{0}\right)$ & $\mathbf{R}_{\mathrm{o}} \mathbf{R}_{\mathrm{f}}$ & $\mathrm{R}_{\mathrm{g}}$ \\
\hline Lait/lait & $\begin{array}{l}\text { I } \\
\text { II }\end{array}$ & $\begin{array}{l}0,87 \pm 0,07 \\
0,83 \pm 0,08\end{array}$ & $\begin{array}{r}1209 \pm 32 \\
944 \pm 32\end{array}$ & $\begin{array}{l}0,80 \\
0,36\end{array}$ & $\begin{array}{l}0,91 \\
0,52 \\
\end{array}$ & $\begin{array}{l}0,88 \\
0,69\end{array}$ \\
\hline $\mathrm{MMU} / \mathrm{MG}$ & $\begin{array}{l}\text { I } \\
\text { II }\end{array}$ & $\begin{array}{l}0,94 \pm 0,08 \\
0,61 \pm 0,07\end{array}$ & $\begin{array}{l}33 \pm 1 \\
25 \pm 1\end{array}$ & $\begin{array}{l}0,78 \\
0,37\end{array}$ & $\begin{array}{l}0,91 \\
0,52\end{array}$ & $\begin{array}{l}0,86 \\
0,71\end{array}$ \\
\hline MTX/TB & $\begin{array}{l}\text { I } \\
\text { II }\end{array}$ & $\begin{array}{l}0,82 \pm 0,05 \\
0,93 \pm 0,09\end{array}$ & $\begin{array}{l}-1,7 \pm 0,1 \\
-1,5 \pm 0,1\end{array}$ & $\begin{array}{l}0,88 \\
0,32\end{array}$ & & \\
\hline $\mathrm{MMU} /(\mathrm{MA}$ et $\mathrm{MG})$ & $\begin{array}{l}\text { I } \\
\text { II }\end{array}$ & $\begin{array}{l}1,08 \pm 0,10 \\
0,63 \pm 0,11 \\
\end{array}$ & $\begin{array}{l}34 \pm 2 \\
27 \pm 1 \\
\end{array}$ & $\begin{array}{l}0,79 \\
0,26 \\
\end{array}$ & $\begin{array}{l}0,86 \\
0,50 \\
\end{array}$ & $\begin{array}{l}0,92 \\
0,52 \\
\end{array}$ \\
\hline $\mathrm{MTX} /(\mathrm{TA}$ et TB $)$ & $\begin{array}{l}\text { I } \\
\text { II }\end{array}$ & $\begin{array}{l}1,15 \pm 0,06 \\
1,45 \pm 0,16\end{array}$ & $\begin{array}{l}-1,6 \pm 0,1 \\
-1,6 \pm 0,2\end{array}$ & $\begin{array}{l}0,93 \\
0,41\end{array}$ & & \\
\hline
\end{tabular}

* Les valeurs génétiques sont, par hypothèse, exprimées en $\mathrm{kg}$ ou g/ $\mathrm{kg}$ et en déviations vis-à-vis des bases 1985 pour les valeurs françaises et 1982 pour celles des Etats-Unis.

Breeding values are, by hypothesis, expressed in terms of $\mathrm{kg}$ or $\mathrm{g} / \mathrm{kg}$ as deviations from the bases of 1985 for French values and 1982 for US values.

Variable, Groupe : voir tableau 1 / see table 1.

$\mathrm{a}, \mathrm{b}, \quad$ : Estimation des paramètres $a$ et $\mathrm{b}$ de la formule de conversion de l'IDF (Amérique du Nord $\rightarrow$ France).

$a$ and $b$ estimated parameters for the IDF conversion formula (North America $\rightarrow$ France).

$\mathrm{r}\left(\mathrm{M}, \hat{\mathrm{G}}_{\mathrm{o}}\right)$ : corrélation entre performances moyennes en France et valeurs génétiques en Amérique du Nord. correlation between French mean deviations and American breeding values.

$\mathbf{R}_{0}^{2} \quad$ : coefficient de détermination des valeurs génétiques en Amérique du Nord. repeatability of breeding values in North America.

$\mathbf{R}_{\mathbf{f}}^{2} \quad$ : coefficient de détermination des valeurs génétiques en France. repeatability of breeding values in France.

$\mathbf{R}_{\mathrm{g}} \quad$ : corrélation génétique entre la France et l'Amérique du Nord. genetic correlation between France and North America.

Les coefficients de régression observés sont homogènes pour le lait $(0,87$ et 0,83$)$. En revanche, il apparaît des discordances importantes entre groupes pour les deux autres critères. Elles ne sont significatives au seuil de $5 \%$ que pour la quantité de 
matière utile. Les valeurs observées pour le coefficient de régression indiquent que dans le groupe II, les écarts de valeur génétique mesurés aux Etats-Unis conduisent pour les filles françaises à des différences plus grandes pour le taux moyen (MTX) et plus faibles pour la quantité de matière utile (MMU) que dans le groupe I. Les moyennes des deux coefficients de régression obtenus sur les deux groupes de données sont égales à 0,85 pour le lait et la quantité de matière utile (MMU), mais sont plus élevées pour le taux moyen $(1,30)$, si MMU et MTX sont prédits avec les matières grasse et azotée. Pour les deux caractères MMU et MTX, les coefficients de régression sont plus élevés lorsque les deux valeurs génétiques de matières grasse et azotée sont utilisées comme prédictrices de la moyenne des performances françaises ; cette augmentation est plus sensible pour le taux de matière utile (MTX).

Les écarts de base entre la France et les Etats-Unis (coefficient a) sont de 1209 ou $944 \mathrm{~kg}$ de lait, de 25 à $34 \mathrm{~kg}$ de MMU selon les estimations. Les écarts sont plus importants dans le groupe I. La prise en compte de la matière azotée augmente légèrement les écarts. En revanche, les résultats sont très concordants pour le taux moyen et une valeur génétique nulle pour le taux aux Etats-Unis semble correspondre à $-1,6 \mathrm{~g} / \mathrm{kg}$ en France.

\section{B. Comparaison avec le Canada}

Les effectifs étant identiques dans les deux groupes de données, la précision d'estimation est nettement meilleure dans le premier groupe de données (taureaux utilisés en France après testage au Canada), qui assure une liaison plus forte entre données canadiennes et françaises (tableau 4).

\section{TABleau 4}

Relations entre les valeurs génétiques * canadiennes et françaises Relationship between breeding values ${ }^{*}$ in Canada and France

\begin{tabular}{|c|c|c|c|c|c|c|}
\hline Variable & Groupe & $\mathrm{b}$ & a & $r\left(M, G_{0}\right)$ & $R_{o} R_{f}$ & $\mathbf{R}_{\mathrm{g}}$ \\
\hline Lait/lait & $\begin{array}{l}\text { I } \\
\text { II }\end{array}$ & $\begin{array}{l}1,14 \pm 0,12 \\
0,45 \pm 0,27\end{array}$ & $\begin{array}{l}524 \pm 61 \\
512 \pm 118\end{array}$ & $\begin{array}{l}0,80 \\
0,22\end{array}$ & $\begin{array}{l}0,84 \\
0,50\end{array}$ & $\begin{array}{l}0,95 \\
0,44\end{array}$ \\
\hline MMU/MG & $\begin{array}{l}\text { I } \\
\text { II }\end{array}$ & $\begin{array}{l}0,84 \pm 0,09 \\
0,77 \pm 0,19\end{array}$ & $\begin{array}{l}15,3 \pm 2,4 \\
18,8 \pm 3,7\end{array}$ & $\begin{array}{l}0,79 \\
0,49\end{array}$ & $\begin{array}{l}0,84 \\
0,50\end{array}$ & $\begin{array}{l}0,94 \\
0,98\end{array}$ \\
\hline MTX/TB & $\begin{array}{l}\text { I } \\
\text { II }\end{array}$ & $\begin{array}{l}0,78 \pm 0,05 \\
0,55 \pm 0,19\end{array}$ & $\begin{array}{l}-0,66 \pm 0,14 \\
-0,62 \pm 0,27\end{array}$ & $\begin{array}{l}0,92 \\
0,38\end{array}$ & & \\
\hline $\mathrm{MMU} /(\mathrm{MA}$ et $\mathrm{MG})$ & $\begin{array}{l}\text { I } \\
\text { II }\end{array}$ & $\begin{array}{l}0,97 \pm 0,10 \\
0,89 \pm 0,23\end{array}$ & $\begin{array}{l}15,8 \pm 2,3 \\
20,1 \pm 4,0\end{array}$ & $\begin{array}{l}0,81 \\
0,48\end{array}$ & $\begin{array}{l}0,83 \\
0,49\end{array}$ & $\begin{array}{l}0,97 \\
0,98\end{array}$ \\
\hline MTX/(TA et TB) & $\begin{array}{l}\text { I } \\
\text { II }\end{array}$ & $\begin{array}{l}1,01 \pm 0,07 \\
0,80 \pm 0,25\end{array}$ & $\begin{array}{l}-0,49 \pm 0,17 \\
-0,57 \pm 0,27\end{array}$ & $\begin{array}{l}0,89 \\
0,42\end{array}$ & & \\
\hline
\end{tabular}

* Les valeurs génétiques sont, par hypothèse, exprimées en $\mathrm{kg}$ ou $\mathrm{g} / \mathrm{kg}$ et en déviations vis-à-vis des bases 1985 pour les valeurs françaises et 1984 pour les canadiennes.

Breeding values are, by hypothesis, expressed in terms of $\mathrm{kg}$ or $\mathrm{g} / \mathrm{kg}$ as deviations from the bases of 1985 for French values and 1984 for the Canadian ones.

Variable, Groupe : voir tableau 1 / see table 1.

$\mathrm{a}, \mathrm{b}, \mathrm{r}\left(\mathbf{M}, \mathrm{G}_{\mathrm{o}}\right), \mathbf{R}_{\mathrm{o}}^{2}, \mathbf{R}_{\mathrm{f}}^{2}, \mathrm{R}_{\mathrm{g}}$ : voir tableau $3 /$ see table 3 . 
Dans le premier groupe de données, les coefficients de régression sont sensiblement proches de 1 sauf dans le cas où seule la matière grasse est utilisée comme variable prédictrice de la matière utile française : le coefficient de régression est alors nettement plus faible, soit 0,84 pour la quantité et 0,78 pour le taux, alors qu'il est proche de 1 dans les deux cas si les matières azotée et grasse sont utilisées simultanément. Le coefficient de régression est nettement supérieur à 1 pour le lait. Les écarts de base sont proches de $15,5 \mathrm{~kg}, 520 \mathrm{~kg}$ et $-0,6 \mathrm{~g} / \mathrm{kg}$ pour la matière utile, le lait et le taux moyen.

Dans le second groupe de données, les coefficients de régression sont tous inférieurs aux valeurs obervées pour le premier groupe. L'écart est très important pour le lait $(0,45$ au lieu de 1,14$)$. Les écarts de base sont plus élevés pour la matière utile (19 ou $20 \mathrm{~kg}$ au lieu de $15,5 \mathrm{~kg}$ ) mais sont très proches, pour les deux autres critères, des valeurs observées dans le premier groupe. Entre les deux groupes, seul l'écart de pentes (b) observé pour le lait est significativement différent au seuil de $5 \%$.

\section{Corrélation génétique entre la France et l'Amérique du Nord}

Les corrélations génétiques estimées sont, pour le premier groupe de données, supérieures à 0,85 pour les Etats-Unis et 0,90 pour le Canada. Dans le second groupe, ces résultats sont plus variables et surtout plus faibles, particulièrement dans le cas des Etats-Unis.

Les corrélations génétiques n'ont pas été calculées pour le taux moyen puisque les publications américaines ne donnent pas de coefficient de répétabilité pour la richesse du lait. Cependant, on peut noter que, dans le groupe I qui offre pour l'estimation de la corrélation génétique une meilleure fiabilité, les corrélations entre les valeurs génétiques américaines et les moyennes françaises excèdent, pour le taux moyen (MTX), d'au moins 0,08 les valeurs obtenues avec les quantités de lait ou de matière utile.

\section{Discussion}

La comparaison des résultats entre les deux groupes de données (I et II) révèle, entre les estimations d'un même coefficient, certains écarts qui ne résultent pas tous de l'imprécision des estimations du groupe II. En particulier, l'écart est significatif et très important pour le coefficient de conversion b de la quantité de matière utile entre les Etats-Unis et la France alors qu'il est faible pour le lait : aucune raison n'est apparue pour expliquer cette différence entre le lait et la matière utile. Le premier groupe semble conduire pour le lait et la quantité de matière utile à des estimations plus élevées pour les coefficients a et $b$. Pour l'expliquer, on peut invoquer le fait que les filles des taureaux du groupe I (semences importées) reçoivent, par divers moyens, un traitement plus favorable que leurs compagnes d'étable, alors que les filles des taureaux du groupe II (taureaux importés) sont issues des programmes de testage et, de ce fait, sont traitées de façon identique à leurs contemporaines d'étable. Si ces traitements préférentiels peuvent expliquer une large part des écarts observés pour le coefficient a, il est douteux qu'ils soient suffisants pour expliquer ceux concernant le coefficient b. L'échantillonnage des mères des taureaux du groupe II peut aussi avoir une influence sur les estimations des paramètres a et b ; malheureusement l'absence d'informations détaillées à ce sujet ne permet de préciser ni l'importance ni le signe d'un éventuel biais d'estimation. Ces résultats sont donc pour certains d'entre eux encore imprécis et quelques incohérences sont observées entre variables. La poursuite de ce travail avec de nouvelles données sera donc utile. 
Malgré cela, des formules de conversion doivent être proposées. En retenant pour les valeurs b, les moyennes des résultats obtenus sur les deux groupes et pour les valeurs a, le groupe II qui est indépendant de traitements préférentiels, on peut convertir les index des Etats-Unis $\left(I_{o}\right)$ exprimés en livres ou pourcentage et relatifs à la demi-valeur génétique du taureau, avec les formules suivantes :

If $=944+(0,85 \times 0,45 \times 2) I_{0}$ pour la quantité de lait

If $=27+(0,85 \times 0,45 \times 2) I_{o}$ pour la quantité de matière utile $(\mathrm{MMU})$

If $=-1,6+(1,30 \times 10 \times 2) I_{o}$ pour le taux moyen $(\mathrm{MTX})$,

les index $I_{o}$ relatifs aux deux variables MMU et MTX étant obtenus par :

$I_{0}=($ Index Matière grasse $+1,15$ Index Matière azotée $) / 2$

$I_{\mathrm{o}}=($ Index Taux butyreux $+1,15$ Index Taux azoté) $/ 2$

Il est plus hasardeux de proposer des formules de conversion pour les index canadiens. Nos estimations sont, faute d'un nombre suffisant de données, moins précises. Aussi est-il intéressant de confronter nos estimations avec les écarts de bases entre les Etats-Unis et le Canada. Après une transformation qui permet d'exprimer les écarts en valeur génétique du taureau et en $\mathrm{kg}$ ou $\mathrm{g} / \mathrm{kg}$, on a entre les Etats-Unis et le Canada :

pour le lait : $786 \times(2 \times 0,45)=707 \mathrm{~kg}$ (Powell, 1985)

ou : $705 \times(2 \times 0,45)=635 \mathrm{~kg}$ (ANONYME, 1984)

pour la MMU : $([18+(1,15 \times 24)] / 2) \times(2 \times 0,45)=21 \mathrm{~kg}$ (Powell, 1985)

pour le MTX : $([-0,04+(1,15 \times[-0,08])] / 2) \times(2 \times 10)=-1,3 \mathrm{~g} / \mathrm{kg}$ (ANONYME, 1984).

Avec les résultats donnés plus haut pour l'écart de base entre la France et les Etats-Unis, ceci conduirait à un écart de base entre la France et le Canada de : 237 ou $309 \mathrm{~kg}$ pour le lait, $7 \mathrm{~kg}$ pour la MMU et $0,3 \mathrm{~g} / \mathrm{kg}$ pour le MTX.

Ces écarts, qui sont, pour le lait et la MMU, nettement inférieurs à nos propres estimations du coefficient a, sont probablement plus précis et doivent être préférés à nos propres estimations. Les estimations obtenues pour le coefficient $b$ sont proches de 1 surtout s'il est possible de connaître la valeur canadienne pour la matière azotée. Néanmoins, ces estimations données pour la conversion entre le Canada et la France restent imprécises et il sera souhaitable de les préciser par l'étude de nouvelles données.

Le groupe II conduit à des corrélations génétiques inférieures à celles du groupe $\mathrm{I}$. Cette incohérence, qui peut s'expliquer en partie par une moindre précision d'estimation dans le groupe II, appelle des études ultérieures qui seront facilitées par le testage actuel de très nombreux taureaux d'origine nord-américaine. Le groupe I conduit à des valeurs plus proche de 1 mais pas suffisamment pour pouvoir conclure à l'absence complète d'interaction génotype-milieu. Lederer (1984), SWANSON (1984), WilminK \& Wismans (1984) observent, dans le cadre de comparaisons entre l'Amérique du Nord et respectivement l'Allemagne, la Grande-Bretagne ou les Pays-Bas des corrélations entre indices de valeur génétique qui sont proches des résultats du groupe I. Il peut donc exister entre les deux continents une petite interaction génotype-milieu. Des différences de niveau de production peuvent en être la cause puisqu'une corrélation génétique égale à 0,86 a déjà été observée en France entre classes extrêmes d'étables (BonAïrt, 1982). Mais, la signification réelle des variables utilisées pour l'évaluation, qui n'est pas la même en France et en Amérique du Nord (système de correction pour la durée de lactation, pondération des différentes lactations, prise en compte des lactations adultes) peut aussi constituer une explication partielle de ces résultats. Des sources de biais conduisant à une sous-estimation de la corrélation génétique ne doivent pas, non plus, 
être négligées. L'estimation de la corrélation génétique dépend de la justesse des coefficients de détermination pris pour la France et l'Amérique du Nord. Certaines valeurs individuelles très élevées de ces coefficients étant peut-être légèrement surévaluées, les corrélations génétiques pourraient être sous-estimées par la méthode utilisée dans cette étude. En outre, les valeurs génétiques estimées en France ou en Amérique du Nord de certains taureaux très populaires peuvent être faussées par des traitements préférentiels qui sont parfois très importants. La sélection des taureaux ou de leurs parents selon les' valeurs génétiques estimées en Amérique du Nord peut aussi conduire à une réduction des corrélations génétiques observées. Il n'est pas possible d'évaluer l'importance de ces biais et d'en tenir compte pour proposer une meilleure estimation de la corrélation génétique. Compte tenu de ces biais et des résultats déjà obtenus entre les Etats-Unis et l'Europe (Lederer, 1984 ; Swanson, 1984 ; Wilmink \& WisMANS, 1984), la corrélation génétique qui est probablement supérieure à 0,85 , ne remet pas en cause l'opportunité des échanges de reproducteurs entre l'Amérique du Nord et la France.

Reçu le 24 octobre 1986. Accepté le 19 février 1987.

\section{Références bibliographiques}

AnONyme, 1984. Use facts and bulls to maximise profit. Int. Newsl. Semex Canada, 3, 1.

BONAïTI B., 1982. Interaction génotype milieu sur la production laitière chez les bovins. II.Corrélation génétique entre niveaux de production et variation de l'héritabilité. Ann. Génét. Sél. Anim., 14, 441-451.

Bonaïti B., Mocquot J.C., Poutous M., 1984. Dairy sire evaluation in France. Int. Dairy Fed. Bull., 183, (Proceedings of the IDF/EAAP Symposium on progeny testing methods in dairy cattle, Prague, September 14-16, 1984), 80-89.

Dairy SiRe Appraisal, 1985. Agriculture Canada-Report 51.

FALCONER D.S., 1952. The problem of environment and selection. Am. Nat., 86, 293-298.

Goddard M., 1985. A method of comparing sires evaluated in different countries. Livest. Prod. Sci., 13, 321-331.

Goursaud J., 1985. Le lait de vache. In : LuQuet F.M. (éd.), Lait et produits laitiers, 1-278. Lavoisier, Paris.

Gravert H.O., 1983. IDF recommended procedure for international comparison of the merit of dairy cattle. Int. Dairy Fed. Bull., 165, 3-6.

LEDERER J., 1984. Estimation of a- and b- values : country report for West-Germany. Int. Dairy Fed. Bull., 183 (Proceedings of the IDF/EAAP Symposium on progeny testing methods in dairy cattle, Prague, September 14-16, 1984), 263-269.

Poutous M., Briend M., Calomiti S., Doan D., Felgines C., Steier G., 1981. Méthode de calcul des index laitiers. Bases générales. Bull. Tech. Ing., 361, 443-446.

Powell R.L., 1985. Converting Canadian sire evaluations to approximate predicted differences. Adv. Anim. Breed., Feb. 1985, 4-6.

Swanson G., 1984. Conversion of bull evaluations from several countries to U.K. improved contemporary comparison equivalents. Int. Dairy Fed. Bull., 183 (Proceedings of the IDF/ EAAP Symposium on progeny testing methods in dairy cattle, Prague, September 14-16, 1984), 293-296.

Wilmink J.B.M., Wismans W.M.G., 1984. A and b- values for the conversion of estimated breeding values for milk production traits in foreign countries to the Netherlands. Int. Dairy Fed. Bull., 183 (Proceedings of the IDF/EAAP Symposium on progeny testing methods in dairy cattle, Prague, September 14-16, 1984), 274-276.

Wilmink J.B.M., Meijering A., Engel B., 1986. Conversion of breeding values for milk from foreign populations. Livest. Prod. Sci., 14, 223-229. 